\title{
Consideration in Microvascular Reconstruction during Times of Social Crises: Perspectives on Resource Utilization
}

\author{
Sean Lloyd Kent, MD ${ }^{1}$ Yohanan Kim, MD ${ }^{1}$ Hector Perez, MD ${ }^{1} \quad$ Ethan Frank, MD ${ }^{1}$ Vance Gentry, BA ${ }^{1}$ \\ Donn LaTour, BA ${ }^{1}$ Jared Inman, $\mathrm{MD}^{1}$ \\ ${ }^{1}$ Department of Otolaryngology, Loma Linda University, Loma Linda, \\ California \\ Address for correspondence Jared Inman, MD, Department of \\ Otolaryngology, Loma Linda University, 11234 Anderson St Suite \\ 2586A, Loma Linda, CA 92354 (e-mail: jared.inman@gmail.com).
}

Facial Plast Surg 2020;36:768-772.

\begin{abstract}
Keywords

- microvascular

- free flap

- head and neck

- pandemic

Reconstruction of head and neck surgical defects can be a complicated, costly process. While the era of cost-effective medicine has begun to broadly question the necessity of high-cost care, times of extraordinary sociomedical demand bring increased scrutiny to even routine costs and resource utilization. Within this context, we reviewed the advantages, drawbacks, and financial costs of both regional and free flap reconstructions, namely the decreased costs and hospital resource utilization that may be associated with reconstruction using regional flaps. Although beset by reports of partial necrosis in certain regional flaps-particularly the submental island, cervicofacial advancement, and supraclavicular artery island flaps-many reports have demonstrated complication and flap failure rates equivalent to those of free flaps. Additionally, regional flaps have been associated with decreased costs for hospital stay, most notably in cases of postoperative complications. In cases necessitating free flap reconstruction, cost-savings strategies such as bypassing postoperative intensive care unit admissions have been shown to provide satisfactory, safe outcomes. As the head and neck surgeon continues to adapt to the medical pressures of a global pandemic, resource-sparing approaches to oncologic care will persist in their newfound importance.
\end{abstract}

For certain defects in the head and neck, free flap with microvascular anastomosis has become standard care at major academic centers. Compared to regional flaps such as pectoralis or cervical facial advancement, free flaps can offer cosmetic and functional advantages. A free flap surgery, however, is costly and typically results in an intensive care unit (ICU) stay of at least one night followed by a hospital stay of 1 week or longer. Given the ongoing coronavirus disease 2019 (COVID-19) pandemic and surging cases particularly affecting the United States, hospital resources, including admissions to the floor and ICU, have come under scrutiny.

\section{Methods}

For this paper, we performed a scope review and summary of literature seeking to understand how hospital resources are managed with respect to free flaps in the head and neck during times of social crises. We explored medical outcomes as well as cost differences between free flaps and regional flaps. We looked at different crises with particular attention to COVID-19 and how head and neck surgery has adapted. Finally, we postulated how future surgical care might be modified during times of resource scarcity.
Issue Theme Technologic Advancements in Head and Neck Reconstruction; Guest Editors: Sameep P. Kadakia, MD, and Sunishka M. Wimalawansa, MD, MBA
Copyright (C) 2020 by Thieme Medical Publishers, Inc., 333 Seventh Avenue, 18th Floor, New York, NY 10001, USA Tel: +1(212) 760-0888.
DOI https://doi.org/ $10.1055 / \mathrm{s}-0040-1721811$. ISSN 0736-6825. 


\section{Outcomes of Regional versus Free Flaps}

Regional flaps present a viable and, in some cases, a preferential alternative to the traditional free flap. A 2019 review article by Jørgensen et al found that procedures utilizing a local flap did not take as long nor did the patients have to stay as long in the hospital as did those involving a free flap. ${ }^{1}$ The advantages and disadvantages of local versus free flaps vary depending on the type of local flap utilized. For this paper, the authors examined five types of regional flaps in the head and neck: submental, pectoralis major, trapezius, cervicofacial, and supraclavicular.

Submental island flaps (SIFs) offer an attractive alternative to free flaps for lower facial surgery repair due to their proximity to the lower face as well as the possibility of eliminating the need for microvascular surgery. SIFs often do not take as long as free flaps ${ }^{1-5}$ and may result in less blood loss ${ }^{2}$ and shortened patient recovery times. ${ }^{1}$

Reports from a number of authors have indicated that patients undergoing SIF surgery have shorter hospital stays than those receiving free flap closure. Jørgensen et al found a pooled mean difference of 2.5 days shorter for SIFs, ${ }^{1}$ Paydarfar and Patel reported SIF closure stays averaging 10.6 days versus 14.0 days for free flaps $(p<0.008),{ }^{3}$ and Howard et al reported 4.9 days for SIF and $9.8(p=0.00817)$ for free flaps. ${ }^{4}$ By switching to a procedure that shortens time in hospital, patients needing urgent cancer resection and reconstruction who would otherwise be denied surgery due to resource scarcity may now be eligible for treatment.

Several potential downsides to local flaps should be taken into consideration when considering therapeutic options. For one, local flap in cancer resection surgery should be used with care and adequate margins to avoid reimplanting cancerous tissue. Within the context of a local submental flap versus a free flap, an SIF can be safely employed in conjunction with a level 1 lymph node dissection provided the lymph nodes are cancer-free. ${ }^{1,6,7}$ Judgment as to whether or not a local flap provides a safe alternative to a remote free flap ultimately is determined by the surgeon.

Amin et al recommend harvesting the submental flap only after completing lymph node dissection and seeking an alternative treatment for patients with nodal disease of $>\mathrm{N} 1 .^{8}$ Elzahaby et al claim no regional nodal recurrence and only $8.3 \%(n=3)$ local recurrence after SIF closure in a cohort in which $33.3 \%(n=12)$ of patients had pathological nodal invasion due to oral squamous cell carcinoma. The authors similarly conclude that SIF closure is acceptable in cases with N0-N1 cancer. ${ }^{7}$ In a 10 -year experience of 50 patients, Howard et al reported success in the use of a SIF with no associated local recurrence. ${ }^{6}$

A second potential downside to local SIFs is their increased propensity for partial flap loss compared to free tissue transfers; however, no significant difference exists with regard to total flap loss. ${ }^{1}$ Based on current literature, a SIF represents an attractive alternative to a free flap closure as long as level 1 lymph nodes are removed with signs of metastasis. $^{6}$
Long considered a staple of reconstructive neck surgery, the pectoralis major flap offers many of the same advantages over free flaps as submental flaps. However, pectoralis flaps are sometimes bulky ${ }^{8-10}$ and limit the reach of the pedicle to sites higher on the head and neck. ${ }^{8}$ The challenge of obtaining appropriate length has been at least partially addressed by Resto et al who described a modified surgical technique resulting in a longer flap, which can then reach up to the lateral skull base. This technique involved harvesting the pectorals major muscle with a skin paddle overlying its complete length. A continuous five centimeter cuff of superior rectus sheath was included and used to suspend the pedicle from lateral pericranium or remaining temporals fascia and muscle. Additionally, the authors recommend cutting motor nerves within the proximal pedicle to prevent strangulation of its vascular supply. ${ }^{11}$ This modified approach offers an alternative to patients not eligible for free flap (e.g., an anterolateral thigh) closure.

For many surgical defects, the pectoralis major flap will remain a feasible alternative to free flaps and offers a high tissue survival rate. ${ }^{10} \mathrm{~A}$ recent study by Spoerl et al reported pectoralis major myocutaneous flap closure as having a slightly lower success rate $(88 \% ; N=40)$ than radial forearm free flap (93\%; $N=230$ ), the same as free fibula flap ( $88 \%$; $N=121$ ) but higher than anterolateral thigh flap closure $(82 \% ; N=51){ }^{12}$ Therefore, even though free flaps may be preferable in typical situations, in times of social crises and limited resources, the pectoralis major flap offers a viable alternative.

The lower trapezius musculocutaneous island flap has somewhat fallen out of favor as it may impair shoulder movement, provide poor aesthetic results, and struggle to reach defects high on the head and neck. ${ }^{4}$ Lower trapezius flaps, however, preserve shoulder function better than lateral and superior trapezius flaps, while providing better aesthetic results. ${ }^{13-15}$ To overcome the problem of limited reach, the lower trapezius flap may be performed by dissecting superiorly beneath the rhomboids to the division of the descending branch of the dorsal scapular artery freeing an extra 2 to $3 \mathrm{~cm}$ of mobility ${ }^{16}$ or by preserving the more superficial transverse cervical artery, which allows for easier rotation of the flap. ${ }^{13}$

Outcomes for lower trapezius musculocutaneous island flaps and muscle-only closure with skin grafts have been reported in small volumes by a number of authors. A 2002 article by Lynch et al reported success in 13 patients undergoing lower trapezius flap closure. ${ }^{13}$ In 1988 , Seyfer reported successful healing in six patients. ${ }^{14}$ A 1997 article by Cole cites one instance of major flap loss in a six-patient experience, ${ }^{16}$ and a 2019 study of six patients documented no instances of major flap loss. ${ }^{17}$

Another attractive alternative to free flaps is the cervicofacial flap. Developed by Juri and Juri, this flap approximates facial color and texture and hides the incisional scar. ${ }^{18,19}$ Traditionally, cervicofacial flaps are used for partial-thickness defects of the temporofrontal region, brow, and orbit, and they remain one of the most common methods of cheek reconstruction. ${ }^{20}$ 
Outcomes for cervicofacial flaps are encouraging. In a 2014 study of 88 patients undergoing cervicofacial flap reconstruction for Mohs ablative surgery by Jacono et al, 24 (27\%) patients recorded some distal end necrosis. Although the authors acknowledge a relatively high rate of reported necrosis (well above what other papers report), they suggest this may be due to their low threshold for defining distal end necrosis, epidermolysis down to $0.25 \mathrm{~cm}^{2}$. Interestingly, of the 88 patients involved, all 23 who experienced distal edge necrosis underwent a superficial cervicofacial flap. ${ }^{20}$ The authors therefore recommend deep plane cervicofacial flaps in particular because they preserve blood flow from the transverse facial artery perforator tissue perfusion better than superficial cervical facial flaps. $^{20}$

Finally, although free flap repair is now the standard of care, the supraclavicular artery island (SAI) rotational flap remains an intriguing option for the reconstruction of head and neck defects. Elucidated by Pallua in the 1990s, and others later on, the use of this flap has been debated over the years because of questions relating to its vascularity and problems with distal flap necrosis. ${ }^{21}$ However, more recent studies comparing the rates of perioperative complications in free flaps versus SAI flaps have proven encouraging. A 2018 systematic review of the literature found that SAI flap reconstruction was faster than free flap reconstruction, and postoperative rates of total flap loss, partial flap necrosis, and recipient donor-site dehiscences were statistically comparable between the two methods. ${ }^{22}$

Compared to free flaps, SAI flaps are relatively quick to harvest (less than an hour), making them ideal in the context of limited operating room (OR) availability. They also approximate many of the aesthetic and functional benefits of free flaps including their thinness, pliability, and similarity to the color of facial skin. ${ }^{23}$ However, SAI flaps do have limitations. Complex three-dimensional defects such as those left by tonsils may be hindered by the rotational nature of the flap. ${ }^{24}$ Additionally, Kokot et al found that SAI flaps longer than $22 \mathrm{~cm}$ were statistically more likely to necrose. ${ }^{24}$

\section{Cost Considerations}

As hospitals strain under decreased revenue due to cancellation of elective cases, costs cannot be ignored. One study conducted from 2015 to 2013 looked at the cost-effectiveness of free flaps for head and neck cancers and found them to be associated with greater quality of life, albeit without any survival benefit. However, the median cost of a free flap without complications was reported to be US\$36,024 compared with the median cost of locoregional reconstruction, which was US\$31,459. Moreover, when factoring in complications, the cost disparity between flap types grows as median cost jumps to US\$67,115 for free flaps and US $\$ 59,512$ for locoregional reconstruction. ${ }^{25}$ In another study specifically comparing the SAI flap to free tissue transfer for the reconstruction of a variety of head and neck defects, the total hospital costs for the pedicled flap were cited as $32 \%$ lower than those of the free flap. ${ }^{26}$
Although head and neck flap failures are relatively rare with an accepted incidence of less than $4 \%$, salvage surgery from these flap complications can significantly increase the overall cost. ${ }^{27}$ The aforementioned Gao et al's study estimates a hospital cost of $\$ 85,761$ in instances of flap failure. ${ }^{25}$ Similarly, in a retrospective review of 438 patients who underwent head and neck microvascular free flap reconstruction between 2005 and 2013 at a single institution, Chang et al note an intraoperative arterial complication rate of $5.5 \%$, which resulted in significantly higher rates of unplanned reoperations, including emergent take-backs and various other major surgical and respiratory complications. These complications increase the total operative time, the length of hospital and ICU, and readmission rates, which translated into a mean hospital cost of US\$80,350 compared with US\$44,803 for patients without arterial complications. $^{28}$

One strategy to mitigate the cost of free flaps would be to obviate the need for ICU stays. Panwar et al compared the outcomes and costs of patients who were postoperatively admitted to the ICU as opposed to a regular unit. The authors found no difference in the rates of flap failure, incidence of reoperation, wound complications, and other pulmonary/cardiac complications between the two groups. However, the non-ICU group did require one less hospitalization day on average, resulting in a comparatively lower cost of US $\$ 28,524$ versus US\$33,642 for the ICU group. ${ }^{29}$ Overall, locoregional flaps cost less compared with free flaps by reducing operative time, shortening hospital stay, and eliminating the need for ICU admission.

\section{Adaptations by Health Care Systems during the Covid-19 Pandemic}

The COVID-19 pandemic has stimulated discussion regarding which types of surgeries should be permitted to continue. Many hospitals have chosen to postpone elective surgeries and nonurgent cases. Given the time-sensitive nature of oncologic surgery and the associated increased risk of operating in the upper respiratory airway tract, otolaryngologists have had to make decisions on appropriate patient candidates in a pandemic setting. Additionally, patients with head and neck tumors may be predisposed to increased severity of infections due to underlying pulmonary disease; thus, surgeries requiring longer inpatient care must be weighed against the risk of patients acquiring COVID-19 while hospitalized.

One hospital in Turin, Italy, a COVID-19 endemic area, had 19 head and neck surgery cases in a 2-month span during the lockdown. ${ }^{30}$ During this period, the hospital only allowed for time-sensitive surgery. Patients underwent preoperative testing, received a chest X-ray, and completed a questionnaire, whereas providers used appropriate personal protective equipment (PPE). Postoperative chest X-ray and COVID19 testing were performed within 24 to 48 hours of hospitalization, with 1 of the 19 patients testing positive for COVID-19. Another institution in Rome, describing changes in surgical activity due to the pandemic, reported limiting 
surgeries to emergencies or oncologic procedures. ${ }^{31}$ An approximate $50 \%$ decrease in total surgical cases was appreciated in the otolaryngology department in comparison to the same period in 2019; however, the amount of oncological cases did not differ significantly between the two time periods.

In Toronto, where the memory of the 2003 SARS (severe acute respiratory syndrome) pandemic remains fresh, an outline of considerations and actions was provided by the Otolaryngology - Head and Neck Surgery Department from the University of Toronto. ${ }^{32}$ Due to the essential role of PPE in combating the spread of the virus and given its relative scarcity, the number of staff allowed in the ORs has been restricted, resulting in limited resident trainee participation in procedures. In cases where surgery was deemed appropriate (advanced cancers, emergencies), a preprocedure discussion was held with the surgical team to confirm that all necessary equipment were available prior to patient arrival. Stronger consideration for regional flaps or delaying reconstruction was given during this time. ${ }^{32}$

Around 35 head and neck oncology organizations have reached an international consensus regarding guidance for proceeding with head and neck oncologic surgery in the pandemic setting. ${ }^{33}$ The recommendations discourage flexible nasoendoscopy without appropriate PPE and recommend that flexible nasoendoscopy should be deferred if the patient does not have a history of head and neck cancer and has lowrisk symptoms, among other statements. An agreement was reached that patients presenting for routine head and neck cancer follow-up may be followed through video or phone conversations. This practice is congruent with the greater increase in the use of telemedicine appointments since the onset of the pandemic to reduce risk of transmission in the outpatient setting. ${ }^{34}$

\section{Conclusion}

As hospital resources are strained, many facial plastic surgeries will inevitably be put on hold. Oncologic surgeries, however, will continue to take priority and need reconstruction. If the hospital and particularly the ICU is at capacity, how will surgeons adapt in a manner to continue providing high-quality care to head and neck cancer patients?

Several possibilities exist. If both a regional and free flap could conceivably cover a defect, a surgeon may elect to perform a regional flap. When only a free flap with microvascular anastomosis will work for a particular defect, the patient may go to a step-down unit with trained staff in lieu of an ICU. One retrospective review of 512 patients who underwent free flap reconstruction of the head and neck at Massachusetts Eye and Ear with postoperative admission to a stepdown unit showed that only $3.5 \%$ of the patients in the series needed a subsequent transfer to the ICU. The authors state that $12.7 \%$ of total cases needed to return to the OR, most commonly for hematoma. Less than a third of the patients who returned to the OR needed anastomotic revision. Whether hourly flap checks in an ICU would have changed these numbers compared with every two hours in a step-down unit is not clear. ${ }^{35}$

Our experience has shown us the necessity of adapting to these new times. This spring, hospital administration called us a few days in advance of a scheduled head and neck reconstruction case involving a free flap to notify us that the procedure had been cancelled due to the ICU being over capacity with COVID-19 patients. Head and neck cancer and reconstructions are time-sensitive and should not be delayed indefinitely. Instead of postponing cases due to a lack of ICU beds, we should consider training intermediate staff in the immediate postoperative care of head and neck cancer patients. The principles of the reconstructive ladder should remain at the forefront of surgical planning; however, during times of crisis, we should consider going down a rung if this change means preserving resources and performing surgery in a timely manner.

\section{Conflict of Interest}

None declared.

\section{References}

1 Jørgensen MG, Tabatabaeifar S, Toyserkani NM, Sørensen JA. Submental island flap versus free flap reconstruction for complex head and neck defects. Otolaryngol Head Neck Surg 2019;161 (06):946-953

2 Aslam-Pervez N, Caldroney SJ, Isaiah A, Lubek JE. A retrospective volume matched analysis of the submental artery island pedicled flap as compared to the forearm free flap: is it a good alternative choice for the reconstruction of defects of the oral cavity and oropharynx? J Oral Maxillofac Surg 2018;76(03):656-663

3 Paydarfar JA, Patel UA. Submental island pedicled flap vs radial forearm free flap for oral reconstruction: comparison of outcomes. Arch Otolaryngol Head Neck Surg 2011;137(01):82-87

4 Howard BE, Nagel TH, Barrs DM, Donald CB, Hayden RE. Reconstruction of lateral skull base defects: a comparison of the submental flap to free and regional flaps. Otolaryngol Head Neck Surg 2016;154(06):1014-1018

5 Patel AV, Thuener JE, Clancy K, Ascha M, Manzoor NF, Zender CA. Submental artery island flap versus free flap reconstruction of lateral facial soft tissue and parotidectomy defects: comparison of outcomes and patient factors. Oral Oncol 2018;78:194-199

6 Howard BE, Nagel TH, Donald CB, Hinni ML, Hayden RE. Oncologic safety of the submental flap for reconstruction in oral cavity malignancies. Otolaryngol Head Neck Surg 2014;150(04): 558-562

7 Elzahaby IA, Roshdy S, Shahatto F, Hussein O. The adequacy of lymph node harvest in concomitant neck block dissection and submental island flap reconstruction for oral squamous cell carcinoma; a case series from a single Egyptian institution. BMC Oral Health 2015;15:80

8 Amin AA, Sakkary MA, Khalil AA, Rifaat MA, Zayed SB. The submental flap for oral cavity reconstruction: extended indications and technical refinements. Head Neck Oncol 2011;3:51

9 Richmon JD, Yarlagadda BB, Wax MK, Patel U, Diaz J, Lin DT. Locoregional and free flap reconstruction of the lateral skull base. Head Neck 2015;37(09):1387-1391

10 McLean JN, Carlson GW, Losken A. The pectoralis major myocutaneous flap revisited: a reliable technique for head and neck reconstruction. Ann Plast Surg 2010;64(05):570-573

11 Resto VA, McKenna MJ, Deschler DG. Pectoralis major flap in composite lateral skull base defect reconstruction. Arch Otolaryngol Head Neck Surg 2007;133(05):490-494 
12 Spoerl S, Schoedel S, Spanier G, et al. A decade of reconstructive surgery: outcome and perspectives of free tissue transfer in the head and neck. Experience of a single center institution. Oral Maxillofac Surg 2020;24(02):173-179

13 Lynch JR, Hansen JE, Chaffoo R, Seyfer AE. The lower trapezius musculocutaneous flap revisited: versatile coverage for complicated wounds to the posterior cervical and occipital regions based on the deep branch of the transverse cervical artery. Plast Reconstr Surg 2002;109(02):444-450

14 Seyfer AE. The lower trapezius flap for recalcitrant wounds of the posterior skull and spine. Ann Plast Surg 1988;20(05):414-418

15 Seyfer AE, Joseph AS. Use of trapezius muscle for closure of complicated upper spinal defects. Neurosurgery 1984;14(03): 341-345

16 Cole I. The lower trapezius island myocutaneous flap for reconstruction of soft tissue of the lateral skullbase and neck. Aust N Z J Surg 1997;67(07):452-456

17 Naalla R, Murthy V, Chauhan S, Chinta K, Singhal M. Revisiting the trapezius flap as a reconstructive option for cervico-occipital and thoracic spine regions. Indian J Plast Surg 2019;52(03):322-323

18 Juri J, Juri C. Advancement and rotation of a large cervicofacial flap for cheek repairs. Plast Reconstr Surg 1979;64(05):692-696

19 Sakellariou A, Salama A. The use of cervicofacial flap in maxillofacial reconstruction. Oral Maxillofac Surg Clin North Am 2014;26 (03):389-400

20 Jacono AA, Rousso JJ, Lavin TJ. Comparing rates of distal edge necrosis in deep-plane vs subcutaneous cervicofacial rotationadvancement flaps for facial cutaneous Mohs defects. JAMA Facial Plast Surg 2014;16(01):31-35

21 Pallua N, Machens HG, Rennekampff O, Becker M, Berger A. The fasciocutaneous supraclavicular artery island flap for releasing postburn mentosternal contractures. Plast Reconstr Surg 1997;99 (07):1878-1884, discussion 1885-1886

22 Sukato DC, Timashpolsky A, Ferzli G, Rosenfeld RM, Gordin EA. Systematic review of supraclavicular artery island flap vs free flap in head and neck reconstruction. Otolaryngol Head Neck Surg 2019;160(02):215-222

23 Chiu ES, Liu PH, Friedlander PL. Supraclavicular artery island flap for head and neck oncologic reconstruction: indications, complications, and outcomes. Plast Reconstr Surg 2009;124(01):115-123

24 Kokot N, Mazhar K, Reder LS, Peng GL, Sinha UK. Use of the supraclavicular artery island flap for reconstruction of cervico- facial defects. Otolaryngol Head Neck Surg 2014;150(02): $222-228$

25 Gao LL, Basta M, Kanchwala SK, Serletti JM, Low DW, Wu LC. Costeffectiveness of microsurgical reconstruction for head and neck defects after oncologic resection. Head Neck 2017;39(03): 541-547

26 Kozin ED, Sethi RK, Herr M, et al. Comparison of perioperative outcomes between the supraclavicular artery island flap and fasciocutaneous free flap. Otolaryngol Head Neck Surg 2016; 154(01):66-72

27 Spiegel JH, Polat JK. Microvascular flap reconstruction by otolaryngologists: prevalence, postoperative care, and monitoring techniques. Laryngoscope 2007;117(03):485-490

28 Chang CS, Chu MW, Nelson JA, et al. Complications and cost analysis of intraoperative arterial complications in head and neck free flap reconstruction. J Reconstr Microsurg 2017;33(05): 318-327

29 Panwar A, Smith R, Lydiatt D, et al. Vascularized tissue transfer in head and neck surgery: is intensive care unit-based management necessary? Laryngoscope 2016;126(01):73-79

30 Riva G, Pizzo C, Fassone E, Pecorari G. Head and neck cancer surgery in COVID-19 pandemic in Northern Italy. Oral Oncol 2020;107:104835

31 Ralli M, Minni A, Candelori F, Cialente F, Greco A, de Vincentiis M. Effects of COVID-19 pandemic on otolaryngology surgery in Italy: the experience of our university hospital. Otolaryngol Head Neck Surg 2020;163(01):86-88

$32 \mathrm{Wu} \mathrm{V}$, Noel CW, Forner D, et al. Considerations for head and neck oncology practices during the coronavirus disease 2019 (COVID19) pandemic: Wuhan and Toronto experience. Head Neck 2020; 42(06):1202-1208

33 Mehanna H, Hardman JC, Shenson JA, et al. Recommendations for head and neck surgical oncology practice in a setting of acute severe resource constraint during the COVID-19 pandemic: an international consensus. Lancet Oncol 2020;21(07):e350-e359

34 Wosik J, Fudim M, Cameron B, et al. Telehealth transformation: COVID-19 and the rise of virtual care. J Am Med Inform Assoc 2020;27(06):957-962

35 Yu PK, Sethi RKV, Rathi V, et al. Postoperative care in an intermediate-level medical unit after head and neck microvascular free flap reconstruction. Laryngoscope Investig Otolaryngol 2018;4 (01):39-42 